Conclusion: In a time of acute crisis, the adaptability of scientifically active female health care workers in rheumatology is lower than that of their male counterparts. This is reflected in a lower scientific output, especially as first or last authors. However, the burden experienced in the current pandemic is similar between the genders.

Disclosure of Interests: None declared

DOI: 10.1136/annrheumdis-2021-eular.3586

\section{POS1469-HPR THE ASSESSMENT OF FRACTURE RISK AND OSTEOPOROSIS RATE AMONG PATIENTS OVER 50 YEARS OLD UNDERGOING MEDICAL REHABILITATION}

\section{Marchenkova ${ }^{1} .{ }^{1}$ National Medical Research Center for Rehabilitation and Balneology of Ministry of Health of Russian Federation, Rehabilitation Department for Somatic Patients, Moscow, Russian Federation}

Background: Taking a course of physical rehabilitation creates the prerequisites for falls and injuries in patients at high risk of fractures. Data on fracture risk and prevalence of osteoporosis in older patients starting medical rehabilitation can change the approach of doctors to the development of rehabilitation programs and the management of such patients.

Objectives: To assess the prevalence of osteoporosis, individual risk factors for osteoporosis as well as the proportion of people with high risk of osteoporotic low-energy fractures among patients over 50 years old undergoing treatment according to the "medical rehabilitation" profile.

Methods: The study group comprised of 600 patients (426 women and 174 men) aged 50 to 84 years, average age $64.25 \pm 10.17$ years, undergoing treatment in a rehabilitation department. This was a cross-sectional study in the form of unified questionnaire, including data concerning age, weight, height, $\mathrm{BMI}$, clinical and rehabilitation diagnosis, anamnesis of the main disease, anamnesis vitae, presence of osteoporosis diagnosis in the anamnesis, its treatment, osteoporosis risk factors estimation. An assessment of 10-year probability of osteoporotic fractures was carried out using Russian model of online FRAX® calculator.

Results: $41.8 \%$ patients in the study sample had osteoporosis risk factors, including $31.2 \%$ of subjects had 3 risk factors or more. $38.0 \%$ patients showed a high fracture risk according to the FRAX calculator. $34.1 \%$ had a diagnosis of osteoporosis, and $45.8 \%$ already had osteoporotic fractures. Among those who did not undergo densitometry examination, $69.9 \%$ had a history of low-traumatic fractures, and only $58.5 \%$ of patients with an established diagnosis of osteoporosis and $26.8 \%$ of those at high risk of fractures received effective therapy for osteoporosis.

Conclusion: Population of patients over 50 years old undergoing rehabilitation is characterized by high frequency of osteoporosis and probability of fractures, and insufficient quality of osteoporosis verification and anti-osteoporotic therapy administration at the same time.

Disclosure of Interests: None declared

DOI: 10.1136/annrheumdis-2021-eular.4258 HPR Interventions (educational, physical, social
and psychological)

\section{POS1470-HPR BARRIERS AND FACILITATORS OF A NEW MODEL OF STRATIFIED EXERCISE THERAPY IN KNEE OSTEOARTHRITIS: A QUALITATIVE STUDY}

J. Knoop ${ }^{1}$, W. Van Berkel-de Joode ${ }^{1}$, H. Brandt ${ }^{1}$, J. Dekker ${ }^{2}$, R. Ostelo ${ }^{1} .{ }^{1}$ Vrije Universiteit Amsterdam, Health Sciences, Amsterdam, Netherlands; ${ }^{2}$ Amsterdam UMC (VUmc), Department of Rehabilitation, Amsterdam, Netherlands

Background: We have developed a model of stratified exercise therapy, in which three knee osteoarthritis $(\mathrm{OA})$ subgroups (i.e., 'high muscle strength subgroup', 'low muscle strength subgroup' and 'obesity subgroup') can be distinguished and provided a subgroup-specific intervention. Currently, the (cost-)effectiveness of this model compared to usual exercise therapy is tested in a large-scaled randomized controlled trial (OCTOPuS-study [1]). Alongside this trial, we performed a qualitative study to explore perceived barriers and facilitators of the application of this model in primary care.

Objectives: To explore barriers and facilitators of the application of this model in primary care, as perceived by patients, physiotherapists and dieticians.

Methods: Qualitative data were collected through semi-structured interviews in a random sample of 15 patients ( 5 from each subgroup), 11 physiotherapists and 5 dieticians, from the experimental arm of the OCTUPuS trial. A thematic analysis of the data was performed.

Results: We identified 14 themes in 5 categories. In general, patients and therapists were positive about the added value and applicability of the model, although some physiotherapists would prefer more flexibility. Regarding the 'high muscle strength subgroup', both patients and physiotherapists reported mixed feelings on the low number of supervised sessions, with some perceiving this low number as advantageous for stimulating the patient's own responsibility, whereas others as hindering an optimally guided treatment. Regarding the 'obesity subgroup' dieticians and physiotherapists acknowledged the added value of the combined intervention, but both were disappointed by the lack of interdisciplinary collaboration. Moreover, those patients in this subgroup already following a diet restriction, therefore not perceiving any added value of the diet intervention.

Conclusion: This qualitative study revealed relevant barriers and facilitators of our new model of stratified exercise therapy, which will help us interpreting the upcoming results on its (cost-) effectiveness [1]. If proven to be (cost-)effective, implementation strategies should specifically focus on guidance of patients from the "high muscle strength subgroup' within only a few sessions, collaboration between physiotherapist and dietician in the 'obesity subgroup', and adequate use of booster sessions after the supervised period to optimize treatment adherence.

\section{REFERENCES:}

[1] Knoop J, Dekker J, van der Leeden M, de Rooij M, Peter WFH, van Bodegom-Vos L, van Dongen JM, Lopuhäa N, Bennell KL, Lems WF, van der Esch M, Vliet Vlieland TPM, Ostelo RWJG. Stratified exercise therapy compared with usual care by physical therapists in patients with knee osteoarthritis: A randomized controlled trial protocol (OCTOPuS study). Physiother Res Int. 2020 Apr;25(2):e1819. doi: 10.1002/pri.1819. Epub 2019 Nov 28.

Disclosure of Interests: None declared

DOI: 10.1136/annrheumdis-2021-eular.1508 